\title{
Mapas del conocimiento, con nombre y apellido
}

\author{
Por Adela d'Alòs-Moner
}

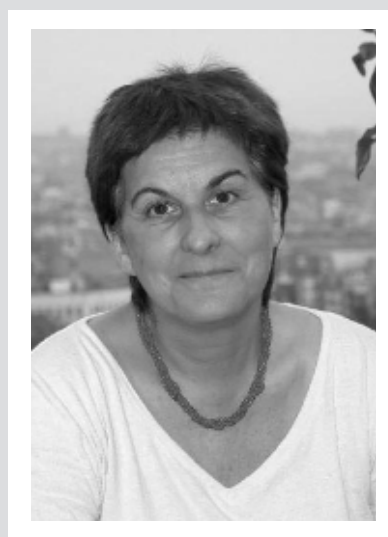

Adela d'Alòs-Moner, socia fundadora de la empresa DOC6 S. A. (1988) - certificada con la ISO 9002 por su sistema de gestión del conocimiento-, realiza trabajos de consultoría en auditorías de la información y del conocimiento. Ha trabajado en la universidad y administraciones públicas como responsable de servicios de información y coordinado los proyectos de la web y la intranet del Ayuntamiento de Barcelona. Coordinadora y evaluadora de proyectos europeos, imparte habitualmente cursos sobre gestión y evaluación de recursos de información, gestión del conocimiento y calidad en los servicios de información.

Resumen: Cuando se audita el conocimiento en las organizaciones se utilizan a menudo mapas del conocimiento para tener una visión gráfica de cuál es la situación de la organización en relación con su conocimiento. En efecto, los mapas permiten identificar y evidenciar las disfunciones y priorizar los aspectos que sería necesario mejorar. Los mapas del conocimiento pueden plasmarse de varias maneras pero, en cualquier caso, es necesario ir más allá de modelos matemáticos o de soluciones informáticas; es indispensable considerar el elemento humano para evitar obtener resultados inútiles o equivocados. No puede hablarse de mapas del conocimiento realmente efectivos en una organización sin tener en cuenta las personas que trabajan en ella, su implicación y su motivación.

Palabras clave: Gestionar el conocimiento, Auditoría del conocimiento, Mapas del conocimiento, Recursos humanos.

\section{Title: Knowledge maps with people behind}

Abstract: When knowledge is audited in organisations, often knowledge maps are used in order to obtain a graphical vision of the situation. Maps allow identifying and making more evident dysfunctions and, consequently, to give priority to the aspects that have to be improved. Knowledge maps can be made in different forms but, in any case, it is necessary to go further of mathematical models or computerised solutions. It is necessary to consider the human element so as to avoid erroneous or useless results. Knowledge maps are inefficient unless employees are fully taken into account, with their implications and motivations.

Keywords: Knowledge management, Knowledge audits, Knowledge maps, Human resources.

EL OBJETIVO DE ESTE ARTÍCULO es explicar las distintas percepciones que existen sobre los mapas de conocimiento y ver cómo contribuyen a centrarse en los conocimientos asociados a los procesos críticos en la organización. La principal conclusión, resultado del análisis de distintas organizaciones, es que detrás de cualquier proceso hay personas y que es indispensable que se produzcan cambios culturales en las organizaciones para poder implantar mejoras en la gestión del conocimiento.

Antonio $G$. ha empezado a trabajar en el centro de documentación de una empresa. Debe atender todo tipo de peticiones de información, tanto de carácter técnico como de marketing o comercial. No domina el sector y además conoce poco la empresa, pero tiene un ins- trumento fundamental para su trabajo. Dispone de unas bases de datos donde encuentra información y conocimiento de gran utilidad. Por ejemplo:

- las preguntas que ha recibido el servicio y las respuestas que se han ido facilitando;

- las fuentes de información que se han utilizado para poder dar la respuesta correcta;

- las personas (técnicos, comerciales o directivos) y su relación con el centro de documentación: desde sus perfiles de interés, hasta su nivel de exigencia, las peticiones que han realizado, el grado de uso del servicio o aspectos a tener en cuenta en el trato con ellos.

Poder disponer de este conocimiento le aporta seguridad y confianza, permitiéndole atender con más eficiencia las peticiones que recibe.

Se le ha informado de la importancia que tiene para la empresa que, una vez que ha dado respuesta a una petición de información, y especialmente si ésta tiene cierta complejidad, anote los recursos que ha utilizado y la percepción que ha tenido en su relación con quien se la ha solicitado.

Maria L. trabaja en una consultoría que desarrolla proyectos tecnológicos para sectores diversos. Su trabajo se ve optimizado gracias a que dispone de un centro de documentación en la empresa que le aporta información sobre proyectos similares, realiza búsquedas, integra y personaliza la información que necesita $\mathrm{y}$, además, ha creado varias bases de datos de recursos con las que sabe: 
- los especialistas o consultores externos a la empresa a los que puede recurrir para que colaboren en un proyecto;

- la relación de estos consultores con anteriores proyectos: calidad del trabajo, nivel de cumplimiento con los plazos de entrega, etc.;

-otros planes similares que se han desarrollado con anterioridad. Disponer de ellos permite reutilizar parte de sus contenidos: estadísticas, datos factuales, gráficos, etc., ya que el centro de documentación ha extraído esta información contenida de los informes, y

—el nivel de satisfacción de los clientes y las sugerencias de mejora que han llevado a cabo con proyectos anteriores.

\section{«Cuando se ha- bla tanto de co- nocimiento co- mo de mapas del conocimien- to, necesaria- mente se debe hablar de perso- nas, con nombre y apellido»}

Tener este conocimiento acumulado le facilita el trabajo y le permite realizar las tareas que le encargan más eficientemente y con más garantías de calidad.
María es consciente de que, una vez finalizado su trabajo, debe invertir un tiempo en introducir su conocimiento basado en la experiencia con el proyecto que ha realizado: relación con los especialistas que han colaborado, comentarios recibidos por el cliente, fuentes de información que le han sido más útiles, etc. El tiempo que debe dedicar le compensa por lo que obtiene de lo que otros han aportado.

En estos dos ejemplos constatamos que existe una información compartida pero, sobre todo, se dispone de un conocimiento compartido que facilita enormemente el trabajo; un conocimiento que alguien ha generado permitiendo que otros lo utilicen y asociado a unos procesos: atender peticiones de información, en el primer caso y desarrollar proyectos en el segundo. Este conocimiento asociado a procesos podemos llamarlo "mapa del conocimiento".

No hay una percepción unívoca sobre qué es un mapa del conocimiento, pero dos son las acepciones más comunes:

-Representación del conocimiento clave que muestra, en cualquier proceso y procedimiento de la organización, la interrelación en el conocimiento que hay en el inicio y al final del proceso. Un ejemplo lo tenemos en la tabla 1 que se comentará con posterioridad.
- Inventario o base de datos del distinto conocimiento existente en la organización, indicando a qué procesos está asociado.

Es importante señalar que el conocimiento de Antonio G. o Maria $L$. no es fruto del azar, sino de la combinación de dos factores esenciales:

a. Que haya una dirección de empresa que impulse una cultura organizacional orientada a potenciar la compartición del conocimiento y el trabajo en equipo. Un ejemplo que se pone a menudo como referencia es el de Nokia. Las iniciativas que se tomaron en el modelo de gestión de la empresa basado en el conocimiento fueron:

- crear la cultura apropiada,

- crear equipos de personas que tuvieran mucho conocimiento, $\mathrm{y}$

- compartir visión, estrategia y nueva cultura de empresa.

b. Que las personas con conocimiento lo compartan; porque "saben que lo que saben" es importante para la organización y son conscientes de que la manera de añadir valor es, precisamente, aportando el propio conocimiento al "saber colectivo". Su actitud debe basarse en la cooperación y en el trabajo en equipo.

En ambos casos es, pues, un conocimiento que alguien introduce en una base de datos, que se usa

\begin{tabular}{|l|l|l|l|l|}
\hline $\begin{array}{c}\text { Suministrador o } \\
\text { proveedor } \\
\text { de conocimiento }\end{array}$ & $\begin{array}{l}\text { Conocimiento que } \\
\text { tienen (inputs de } \\
\text { conocimiento) }\end{array}$ & \multicolumn{1}{|c|}{ Proceso clave } & $\begin{array}{c}\text { Conocimiento que generan } \\
\text { (output de conocimiento) }\end{array}$ & $\begin{array}{c}\text { Departamento (o cliente } \\
\text { interno) }\end{array}$ \\
\hline Ventas & $\begin{array}{l}\text { Informe visita a clientes } \\
\text { (percepción de } \\
\text { requerimientos del } \\
\text { cliente) }\end{array}$ & $\begin{array}{l}\text { Adecuación a los } \\
\text { requerimientos de los clientes }\end{array}$ & Especificaciones de productos & Servicio de I\&D \\
\hline Dep. técnico & $\begin{array}{l}\text { Relación con técnicos } \\
\text { subcontratados } \\
\text { (rigor, capacidad de } \\
\text { trabajo, puntualidad, } \\
\text { etc.) }\end{array}$ & Desarrollo de productos & Selección de personal técnico & Recursos humanos \\
\hline
\end{tabular}


y reutiliza. Es por ello que, cuando se habla de tanto de conocimiento como de mapas del conocimiento, necesariamente se debe hablar de personas, con nombre y apellido. Son las personas, sus actitudes y aptitudes, las que realmente hacen que un mapa del conocimiento sea algo más que un diagrama o un modelo conceptual sobre papel y se convierta en una herramienta que pueda realmente representar una ventaja competitiva para la empresa. La sociedad de la información sitúa la información y el conocimiento como un elemento central y factor diferencial. Es por ello que las personas y su conocimiento son un elemento clave para las organizaciones.

\section{«Los mapas del conocimiento permiten tener una visión gráfi- ca de cuál es la situación de la organización en relación con su conocimiento, entendido como parte de su capi- tal intelectual»}

Ya en 1969 Peter Drucker citó, por primera vez, el término "knowledge worker" para designar a las personas o trabajadores que aportan conocimiento en las organizaciones. Esta relación biunívoca entre personas y conocimiento hace que el número de "trabajadores del conocimiento" aumente. Otros autores consideran que todos los empleados deben ser tratados como "trabajadores del conocimiento" ya que las empresas son, a la vez que instituciones económicas, repositorios de conocimiento.

Gestionar hoy una institución pasa, en gran medida, por gestionar estos "trabajadores del conocimiento":
-Cómo se accede al conocimiento tácito que tienen los empleados.

-Cómo se motiva, se incentiva el compromiso y la identificación con los valores de la organización.

-Cómo se consigue que los expertos, los profesionales (que a menudo se constata que son los más resistentes al cambio) se involucren y acepten los cambios.

-Cómo se gestiona el tiempo de los expertos, ya que gestionar el conocimiento comporta siempre una dedicación a veces difícil de manejar.

-Y, sobre todo, cómo se cambia la cultura basada en "saber es poder".

La motivación y la creatividad de las personas aparecen como ideas clave. Amabile (1998) señala seis factores para potenciarlas:

-Ofrecer nuevos retos.

-Permitir un nivel de libertad. dinero).

-Facilitar recursos (tiempo y

- Potenciar el trabajo en equipo.

-Que la organización en su conjunto dé soporte.

-Una supervisión, desde la dirección, que estimule y potencie las ideas.

Con todo ello llegamos a una pregunta que no es fácil de responder ¿qué es el conocimiento? Aunque hay distintos tipos de clasificaciones (saber qué, saber cómo, saber dónde, etc.) los dos que habitualmente aparecen con más frecuencia son el conocimiento explícito y el conocimiento tácito. Por el primero se entiende aquel que puede expresarse en palabras o números y se puede comunicar fácilmente. Por tácito el que es personal, específico en contexto, difícil de formalizar y de comunicar. Ges- tionarlo comporta una visión dinámica de ambos (tácito y explícito) $\mathrm{y}$, en este aspecto, dos autores, (Nonaka; Takeuchi, 1995) diseñaron el modelo que ayuda a explicar cómo se pasa de uno a otro: Seci (socialización / externalización / combinación / internalización).

«Es necesario
asegurar que el
conocimiento
asociado a los
procesos clave
de la organiza-
ción se captura
(y se usa) de
manera sistemá-
tica»

Nonaka (1991) dice que la creación de nuevo conocimiento depende de la visión, percepción, intuición personal y que, para ello, es clave el compromiso de las personas que trabajan en la organización. Habla también del papel vital que tienen los equipos en la creación de conocimiento y el rol de los cuadros directivos. Su modelo responde a la cultura de empresa en Japón donde el trabajador se identifica con la empresa y ésta entra a formar parte de su familia. La base es el $b a$, plaza o lugar de encuentro, ya que para los japoneses las relaciones de trabajo en las organizaciones tienen una enorme importancia.

El modelo occidental cuestiona en gran medida el anterior y defiende la idea de que el conocimiento no puede ser fácilmente detectado, expresado en lenguajes o codificado y, por tanto, compartido. Únicamente tiene sentido en un contexto determinado y es difícil de codificar. Esta visión privilegia el pensamiento individual y objetivo, contrastando con el énfasis en Japón de potenciar el conocimiento tácito y colectivo. 
Si sobre ¿qué es el conocimiento? ya hay distintas percepciones, sobre gestionarlo encontramos múltiples definiciones. Posiblemente una de las más completas la facilita Skyrme: "es la gestión explícita y sistemática del conocimiento vital y está asociado con el proceso de creación, organización, difusión, uso y explotación". Aparecen aquí cuatro conceptos clave:

-Explícito. Nos indica que el conocimiento está considerado como un bien, valor clave en la organización, potenciado por su dirección, validado por su modelo organizativo.

- Sistemático. La sistematización nos asegura que las personas y los equipos están preparados (técnica y culturalmente) para enriquecer el bien común, usarlo, reutilizarlo y eliminarlo si es conveniente ya que, tan importante como capturar y almacenar memoria corporativa y conocimiento crítico, es que haya una "cultura de la destrucción" que elimine lo viejo, lo que no sirve.

-Vital. Nos confirma que el conocimiento a gestionar estará asociado a los objetivos de la organización y a los procedimientos clave del negocio.

-Procesos. Deben asegurar que los que tienen que aportar puedan hacerlo con facilidad y los que tienen que usar el conocimiento común puedan explotarlo fácilmente y traducirlo en nuevo conocimiento enriquecido con la propia experiencia. Un conocimiento accesible en el momento justo y para quien lo necesita.

El mapa del conocimiento facilita identificar las disfunciones existentes entre la situación deseada y la actual:

- ¿Hay un responsable de asegurar que el conocimiento necesario asociado a un proceso se hace explícito a quién lo necesita?
- ¿Se captura el conocimiento de manera sistemática?

—Es fácil acceder a este conocimiento?, ¿cómo se llega a él?

- ¿En qué formato está disponible: base de datos, papel, etc.?

_ ¿Cómo se usa?

— ¿Cómo se asegura la calidad y la validez de los contenidos?

Los mapas del conocimiento hacen posible tener una visión gráfica de cuál es la situación de la organización en relación con su conocimiento, entendido como parte de su capital intelectual. Permiten identificar y plasmar las disfunciones y priorizar los aspectos que sería necesario mejorar con el objetivo de minimizar riesgos, ahorrar costes o mejorar el servicio al cliente. El objetivo final es claro: hay que asegurar que el conocimiento asociado a los procesos clave de la organización se captura (y se usa) de manera sistemática.

\section{«Las líneas de actuación de fu- turo deberían ir dirigidas más a cambios de cul- tura organizacio- nal que a imple- mentaciones tecnológicas»}

Estos mapas son, en general, el resultado de una auditoría del conocimiento. A diferencia de las auditorías de la información, que contemplan sobre todo aspectos más tangibles, capturables y fácilmente transmitibles, evalúan aspectos menos identificables como la experiencia o el "saber hacer". El conocimiento es más intangible, más personal y menos codificable. Para construir un mapa del conocimiento es importante realizar dos procesos:

-Una auditoría del conocimiento, que empieza por entender la organización, su estrategia, sus objetivos y prioridades, su evolución y sus necesidades de información y de conocimiento. Incluye también, una evaluación de las nuevas aptitudes que la empresa demanda de las personas que trabajan en ella, teniendo en cuenta el contexto tan cambiante.

- Identificar los procesos clave de la organización y entender cuáles son los inputs de información y de conocimiento que se necesitan para llevarlos a cabo en las mejores condiciones.

El mapa deberá contener:

-Los procesos y las actividades clave.

—La información y el conocimiento estratégico que se necesita para llevarlas a cabo.

—Quiénes son las personas (clientes/usuarios internos o externos) asociados y quienes son los suministradores o proveedores de información y conocimiento. $\mathrm{O}$, lo que es lo mismo, dónde están las preguntas y dónde las respuestas que la institución necesita para realizar sus procesos de manera más efectiva.

- Cómo usan las personas la información y el conocimiento.

Como ejemplo, para simular un mapa del conocimiento y siguiendo unas de las dos acepciones indicadas más arriba, podemos pensar en una empresa de tecnología de la información, donde dos de sus procesos clave a considerar son la adecuación de los programas informáticos que realizan según los requerimientos de los clientes y la innovación, con el desarrollo de nuevos productos. En estos dos procesos tenemos unos proveedores que actúan como suministradores de información y conocimiento (los que tienen las respuestas) y unos clientes que necesitarían la información para realizar sus tra- 
bajo en mejores condiciones (los que poseen las preguntas).

Un posible esquema sería el siguiente (tabla 1):

-En el centro tenemos identificados dos procesos clave: adecuación a los requerimientos de los clientes y desarrollo de nuevos productos.

- A la izquierda se encuentran los suministradores o proveedores que acumulan, a través de su relación con el cliente en un caso, o con técnicos externos contratados en el otro, un importante conocimiento.

- A la derecha, los departamentos dentro de la organización (o clientes internos) para los que la información y conocimiento que tienen los departamentos anteriores (en nuestro caso el de ventas y el técnico) es fundamental para poder realizar su trabajo con la máxima eficiencia. Se establece una relación dinámica entre los "inputs" (conocimiento que se aporta) y los "outputs" de conocimiento (resultado del acceso y uso del conocimiento aportado).

En ambos casos, en los dos ejemplos anteriores, vemos que no se trata de información, sino de conocimiento asociado a unos procesos. En un caso es un conocimiento que tiene el personal de venta, en el otro, el departamento técnico. En los dos procesos hay un conocimiento asociado (sobre los requerimientos de los clientes o sobre personas contratadas para desarrollos específicos) que es de gran importancia para la organización, ya que están asociados a procesos clave.

\section{Unas conclusiones}

Los mapas del conocimiento pueden expresarse de varias maneras y se han descrito modelos matemáticos para poder construir sistemas que plasman el conocimiento necesario, el disponible y las personas o equipos que lo tienen $\mathrm{y}$ el que necesita la organización. También se han traducido a menudo en la implementación de herramientas informáticas que hacen de repositorio.

Quedarse aquí, en simples modelos matemáticos o en soluciones informáticas, sin considerar el elemento humano puede llevar a resultados inútiles o equivocados. Un caso evidente es el de las intranets. Mientras hay un reconocimiento generalizado de las grandes posibilidades que ofrecen, también se constata a menudo que muchas de ellas no han ayudado a compartir el conocimiento. Si bien han servido para compartir datos o información, no han sido lo suficientemente efectivas para compartir el conocimiento.

Los motivos son siempre los mismos, la historia se repite: o bien la dirección de las empresas no ha sabido ver la importancia de compartir el conocimiento y, por tanto, no ha creado la cultura necesaria ni tampoco se ha potenciado, o bien las personas que deben aportar lo que saben, no lo hacen o no lo realizan de manera sistemática.

Las líneas de actuación de futuro deberían, pues, ir dirigidas más a cambios de cultura organizacional que a implementaciones tecnológicas. La cultura de la empresa y sobre todo el liderazgo claro de la dirección y la implicación de cada uno de sus trabajadores, es el elemento clave.

Manuales de procesos o procedimientos, la intranet, aplicaciones informáticas, etc., pueden ayudar, pero no puede hablarse de mapas del conocimiento efectivos en la organización sin el compromiso personal, si no existen personas implicadas, motivadas, con nombre y apellido.

\section{Bibliografía}

Amabile, T. "How to kill creativity". En: Harvard business review, 1998, sep-oct, pp. 77-87.
Drucker, P. The age of discontinuity: guidelines to our changing society. London: Heinemann, 1969.

Gordon, E. E. "The new knowledge worker". En: Adult learning, 1997, v. 8, n. 4, pp. 14-18.

Gordon, J. L. "Creating knowledge maps by exploiting dependent relationship". En: Knowledge-based systems, 2000, n. 13, pp. 71-79.

"Knowledge maps: an essential technique for conceptualisation". En: Data and knowledege engineering, 2000, n. 33, pp. 169-190.

Nonaka, I; Takeuchi, H. The knowledge-creating company. Oxford: Oxford University Press, 1995.

Skyrme, D. J. Creating the knowledge based business. London: Business Intelligence Ltd., 1997.

Stanford, X. "Map your knowledge strategy". En: Information outlook, 2001, June, v. 5, n. 6, pp. 18-28.

Adela d'Alòs-Moner, DOC6. aalos@doc6.es 